\title{
Deutsch-türkisches Code-Switching und Code-Mixing in einer Gruppe von akademischen Migranten der zweiten Generation
}

\begin{abstract}
Dieser Beitrag geht zurück auf meine 2010 erschienene Dissertation „Migration, Sprache und Rassismus“. Dort untersuche ich mit ethnografischen und gesprächsanalytischen bzw. -rhetorischen Methoden den kommunikativen Stil von zwei mehrheitlich türkischstämmigen, akademischen Gruppen der zweiten Migrantengeneration in Deutschland. Im Fokus der Studie steht das Milieu der „emanzipatorischen Migranten“, die ethnische Zuschreibungen wie „Türken“, „Kurden“, Italiener“" etc. ablehnen und sich als (kultur-)politische Initiativen gegen Diskriminierung und für gleiche Bürgerrechte einsetzen. ${ }^{1}$ Als Fallstudie für diese soziale Welt analysiere ich die Mannheimer Gruppe der „Unmündigen“. Mein Erkenntnisinteresse richtet sich auf die Fragen, welche rhetorischen Verfahren die Beteiligten im Umgang mit Diskriminierungs- und Marginalisierungserfahrungen entwickelt haben und wie sie in ihrer gruppeninternen Kommunikation auf das Türkische und das Deutsche in ihrem Sprachrepertoire zurückgreifen. Im vorliegenden Artikel werde ich auf den letztgenannten Aspekt eingehen und untersuchen, welche Formen und Funktionen die deutsch-türkische Variationspraxis der „emanzipatorischen Migranten“ aufweist.
\end{abstract}

\section{Untersuchungen zur Sprachvariation}

Die Untersuchung von Sprachwahl- und Variationsaspekten hat in der Soziolinguistik eine lange Tradition. Seit der Etablierung der Disziplin vor sechs Jahrzehnten haben verschiedene Forschungsansätze Erkenntnisse von unterschiedlicher Reichweite an den Tag gefördert: a) Sprachsoziologische Arbeiten fokussieren mit Konzepten wie „Diglossie“ (Ferguson 1959) oder „Domäne“ (Fishman 1965) auf der Makroebene die gesellschaftliche Mehrsprachigkeit; b) anthropologische Ansätze in der Tradition der „Ethnografie der Kommunikation“" (Gumperz/Hymes (Hg.) 1964, (Hg.) 1972) untersuchen Kommunikations- und Variationsstrukturen auf der gesellschaftlichen Mesoebene wie in kleinen Gemeinden oder sozialen Gruppen; und c) schließlich wird auf der Mikroebene mit dem konversationsanalytischen Ansatz (Auer 1984) die Verwobenheit der Sprachwahl und Variationsaspekte mit sequenziellen Interaktionsstrukturen analysiert. Für meine Untersuchung

Die zweite soziale Welt der ,akademischen Europatürken“, die ich in meiner Dissertation als Kontrastmilieu zu den „emanzipatorischen Migranten“ untersuche, identifiziert sich ethnisch als in Europa/Deutschland lebende Türken. Zum kommunikativen Sozialstil dieser Gruppe, auf die ich im vorliegenden Aufsatz nicht weiter eingehe, siehe in Cindark (2010). 
der Variationspraxis einer sozialen Gruppe sind die beiden Ansätze von Gumperz und Auer von Relevanz, auf die ich im Folgenden näher eingehen möchte.

Gumperz zählt mit seinen zahlreichen ethnografischen Feldstudien zu den wichtigsten Initiatoren der interpretativen und interaktionalen Soziolinguistik. Insbesondere mit seiner Arbeit zur Sprachvariation in der norwegischen Stadt Hemnesberget, die er Anfang der 1970er mit Blom vorstellte, hat er innerhalb der Soziolinguistik das Paradigma der „Code-SwitchingForschung" (mit-)konstituiert. Die Studie ist die Pionierarbeit, die a) die Sprachvariation als kreative Hervorbringung von Interaktionsbeteiligten und b) die Bedeutung von Sprachvariationsmustern als Ausdruck der sozialen Orientierung von Sprechern unterstreicht. In ihrer Arbeit halten Blom/ Gumperz (1972) zunächst fest, dass alle Bewohner der Gemeinde in ihrem linguistischen Repertoire ${ }^{2}$ mehr oder weniger über zwei Codes verfügen: den lokalen Dialekt Ranamål und die norwegische Standardsprache Bokmål. Die Analyse von verschiedenen Gruppengesprächen zeigt jedoch, dass Sprecher mit unterschiedlicher sozialer Orientierung die Codes in der Interaktion auch unterschiedlich verwenden. Die Ortsloyalen sprechen in der gruppeninternen Kommunikation fast durchgängig Dialekt, mit nur einigen wenigen lexikalischen Elementen aus der Standardsprache (Blom/Gumperz 1972, S. 431). Eine Gruppe von Studenten, die sich seit einigen Jahren nur in den Semesterferien im Ort aufhalten und sich sozial durch eine bidirektionale Orientierung auszeichnen (Ortsloyalität und Loyalität gegenüber pan-norwegischen Werten), zeigt auch in ihrer gruppeninternen Kommunikation ein häufiges Wechseln zwischen Dialekt und Standard. Schließlich erfassen die Autoren eine dritte lokale Gruppe, deren Orientierung außerhalb der Kleinstadt auf die städtische Mittelschicht gerichtet ist, die in der gruppeninternen Kommunikation die Standardvarietät sprechen und Dialekt nur für besondere kommunikative Effekte verwenden, wie etwa bei Erzählungen ortsbezogener Anekdoten (ebd., S. 434).

Die Studie von Blom/Gumperz wurde in der Folgezeit häufig rezipiert, was vor allem an zwei Beschreibungskategorien lag, die die Autoren zur Einordnung der Sprachalternationsmuster entwickelt haben: „metaphorisches" und ,situatives Code-Switching“. Mit ,situativem Code-Switching“ beschreiben Blom/Gumperz die Variationsfälle, die eine Änderung der Situationsdefinition indizieren. Dagegen erfassen die Autoren mit dem Konzept des ,metaphorischen Code-Switchings“ all die Sprachalternationen, die im Rahmen eines Aktivitätskomplexes vorkommen und somit keine Ände-

\footnotetext{
$2 \quad$ Nach Gumperz (1972, S. 20) umfasst das linguistische Repertoire die ,totality of linguistic resources (i.e., including both invariant forms and variables) available to members of particular communities“. Das Konzept wurde in den 1960ern im Rahmen anthropologischer Feldstudien entwickelt als Antwort auf die Feststellung, dass „, the linguist's grammatical analysis seldom matches verbal behavior of actual populations" (ebd.).
} 
rung der Situationsdefinition auslösen. ${ }^{3}$ Diese Perspektive auf Sprachalternation als eine interaktiv und sozial bedeutsame Kommunikationspraxis stellte zum damaligen Zeitpunkt eine ganz neue Betrachtungsweise des Gegenstands dar. Zuvor wurde in Bezug auf Code-Switching häufig von einem idealen bilingualen Sprecher ausgegangen (Weinreich 1953) oder man ging so weit, das Wechseln zwischen Sprachen bzw. Sprachlagen als eine „unregelmäßige Mixtur“ (Labov 1971) ohne jede Systematik zu betrachten, wie aus den folgenden zwei Zitaten hervorgeht:

The ideal bilingual changes from one language to the other according to appropriate changes in the speech situation (interlocutor, topic etc.), but not in an unchanged speech situation and certainly not within a single speech situation. (Weinreich 1953, S. 73)

In fact, bilingual speakers do produce strange mixtures of the two languages, [...]. So far, however, no one has been able to show that such rapid alternation is governed by any systematic rules or constrains, and we therefore must describe it as the irregular mixture of two distinct systems. (Labov 1971, S. 457)

Bezogen auf das letzte Zitat von Labov konnten also Blom/Gumperz in ihrer Studie aufzeigen, dass das Alternieren zwischen Sprachen bzw. Sprachlagen nicht als eine „eigenartige“ bzw. „unregelmäßige Mixtur“ aufzufassen ist, sondern spezifische Formen der Variation eine ganz bestimmte soziale Orientierung der Sprecher zum Ausdruck bringen (kann). In der Folge arbeitete Gumperz (1982) seinen interpretativ-soziolinguistischen Ansatz zur Sprachvariation weiter aus, indem er neben den gruppenspezifischen Funktionen des Code-Switchings a) auf der Ebene des Diskurses verschiedene Funktionen von Sprachalternationen beschrieb und b) seine Theorie der Kontextualisierung entwickelte.

Bei der Kontextualisierungstheorie geht Gumperz (ebd., S. 130 ff.) davon aus, dass Makro- oder Mikrokontexte von sozialen Situationen nicht einfach gegeben sind, sondern von den Akteuren hergestellt, bestätigt oder auch verändert werden. Bei Gesprächen zeigen die Beteiligten in lokalsequenziellen Äußerungen bis hin zu - zum Teil relativ festgelegten - situativen Rollen jederzeit an, in welchem Sinne, d.h. in welchem Kontext ihre Äußerungen und ihre Beteiligungsweisen zu verstehen sind. Das Indizieren von Kontextbezügen stellen sie durch Hinweise dar, die sowohl verbale als auch nonverbale Elemente umfassen. Als eine der verbalen Kontextualisierungsmittel bezeichnet Gumperz das Wechseln zwischen Sprachen und Sprachlagen. Mit anderen Worten können die Beteiligten mit Hilfe des Kontrasts, der durch das Wechseln von einem linguistischen Code zum anderen entsteht, anzeigen, in welchem Kontext ihre Äußerung zu verstehen

In seiner später erschienenen und einflussreichen Monografie „Discurse Strategie““ (1982) spricht Gumperz dann nicht mehr von „metaphorischem“ sondern „konversationellem“ Code-Switching. 
ist. In dem Sinne spricht er davon, dass durch einen Codewechsel eine Änderung von Interaktionsrollen, Modalitäten, Themen etc. kontextualisiert wird bzw. werden kann. ${ }^{4}$

Auer (1984) hat im Folgenden die Untersuchung von Sprachwechsel als Kontextualisierungshinweise aufgenommen und weiterentwickelt, indem er bei der Untersuchung bilingualer Interaktionen als erster die Methoden und Konzepte der Konversationsanalyse anwandte. ${ }^{5}$ Bis zu diesem Zeitpunkt wurde auch in der interpretativen Soziolinguistik Code-Switching nicht unter dem Gesichtspunkt analysiert, dass es als kommunikative Praxis mit anderen Ebenen der Interaktionsstruktur in einer Wechselwirkung steht. ${ }^{6}$ Die wesentliche Leistung des Ansatzes ist, dass er einzelne Variationsfälle interaktionsanalytisch in ihrem sequenziellen Kontext untersucht und Sprachalternation im Gespräch nicht ausgehend von außersprachlichen Faktoren erklärt. Somit können mit Hilfe der konversationsanalytischen Methode häufig nur postulierte Konzepte wie „they-code“ und „we-code“ (Gumperz 1982) oder Maximen wie „Variation als markiertes und unmarkiertes Sprechen" (Myers-Scotton 1993b), wenn sie denn in einer Interaktion wirksam sind, konkret erfasst und nachgewiesen werden.

Des Weiteren hat Auer (1999) eine Typologie des bilingualen Sprechens vorgelegt, die die vielfältigen Sprachalternationserscheinungen auf einem Kontinuum verortet und drei prototypische Ausprägungen von Kontaktphänomenen beinhaltet: „,code-switching“ (CS), „language mixing“ (LM) und ,fused lects“ (FL). Unter Code-Switching subsummiert Auer die Alternationsfälle, bei denen die Juxtaposition von zwei Codes (Sprachen, Dialekten, Varietäten etc.) von Interaktionsteilnehmern als ein sequenziell bedeutungsvolles Ereignis angesehen wird. Die prototypischen Fälle von Code-Switching erscheinen in Interaktionen und soziolinguistischen Kontexten, in denen die Sprecher der Präferenz ,eine Sprache zu einer Zeit“ (ebd., S. 311 f.) folgen. Aufgrund dieser Präferenz ist es möglich, vor dem Auftreten des Wechsels einen bestimmten Interaktionscode zu identifizieren. Ausgehend vom etablierten Code der Interaktion signalisiert dann das Code-Switching „Andersartigkeit" (ebd., S. 312) der neuen kontextuellen Rahmung. Nach Auer kann man das belegen, indem gezeigt wird, ,that

\footnotetext{
Zum Konzept der Kontextualisierung siehe u.a. Auer (1986) und Schmitt (1993).

So hebt Auer (1984, S. 24) hervor, dass Code-Switching ,,concerns the organization of conversation, that is, turn-taking, topical cohesion, tying, sequencing of activities, repair, overall organisation, etc.“. In dem Sinne analysiert er u.a. Sprachwechsel zur Bearbeitung von „double cohesion“ (ebd., S. 42 ff.) oder zur Anzeige von „footing“-Wechsel (ebd., S. 13 ff.).

6 Der von Auer entwickelte konversationsanalytische Zugang zu bilingualer Kommunikation wurde in der Folge in Bezug auf verschiedene Mehrsprachigkeitskontexte angewandt. Siehe z.B. in Wei (1998) und Sebba/Wootton (1998). Keim (1995) hat die Konzepte und Methoden von Gumperz und Auer in Bezug auf die Analyse von Standard-Dialekt-Daten angewandt.
} 
[juxtaposition] is used as contextualization cue (i.e., that it is ,functional ${ }^{l}$ " (ebd.). ${ }^{7}$ Als Kontextualisierungshinweis indexikalisiert der Wechsel nach Auer entweder Aspekte der Interaktion („,diskursbezogenes Code-Switching“) oder der Sprecher (,teilnehmerbezogenes Code-Switching“).

Als Code-Mixing fasst Auer dagegen die Variationspraktiken, bei denen die Verwendung von Elementen zweier (oder mehrerer) Codes für die Teilnehmer nicht mehr sequenziell sondern in einem sozialen und globalen Sinne bedeutsam ist, zum Beispiel als Gruppencode. Aufgrund der „,numerous and frequent cases of alternation" geht dabei das Potenzial des CodeSwitchings als Kontextualisierungsstrategie verloren, sodass die einzelnen Juxtapositionsfälle beim Code-Mixing „do not carry meaning qua language choice for the bilingual participants“ (Auer 1998, S. 16). Diese Variationspraxis zeichnet sich dadurch aus, dass man nur schwer oder unmöglich einen Code bzw. eine Sprache als den Interaktionscode identifizieren kann. Nicht eine der beteiligten Sprachen, sondern ihr alternierender Gebrauch an sich ist als Interaktionscode selbst anzusehen. Besondere Beachtung schenkt Auer in diesem Kontext der Übergangsphase von Switching zu Mixing. Zu den ersten darin beobachtbaren Phänomenen zählt er die Fälle von lokal bedeutungsvollem Code-Switching, die in diskursbezogener Funktion eingesetzt werden, aber keinen Wechsel der Interaktionssprache darstellen, wie zum Beispiel kleinräumige Wechsel zur Markierung von neuen Gesprächsthemen. Andere Fälle sind für Auer die frequente Verwendung von „(nonce) borrowings" statt funktionalen Insertionen und Diskursmarkern (ebd., S. $17 \mathrm{ff}$.). Außerdem ist nach Auer der Übergang eine Phase, in der ,the ,older' CS [Code-Switching] pattern and the ,newer' LM [Language Mixing] pattern co-exist" (ebd., S. 10), weshalb er darauf hinweist, dass ,in a given conversation [...] it is analytically difficult to disentangle the two phenomena" (ebd., S. 16).

\section{Eine Fallstudie zu den „emanzipatorischen Migranten“}

Ab Mitte der 1980er und verstärkt zu Beginn der 1990er Jahre gründeten sich in Deutschland Migrantengruppen mit Namen wie „Kauderzanca“ in Berlin, „Saz-Rock“ in Frankfurt, „köXüz“ in Hamburg oder „Die Unmündigen“ in Mannheim. Ihre Akteure waren Kinder der so genannten „Gastarbeiter", d.h. Angehörige der zweiten Migrantengeneration, die in Deutschland geboren wurden bzw. aufgewachsen sind, und die sich in jener Zeit als Jugendliche bzw. junge Erwachsene für gesellschaftspolitische Fragen zu

Eine andere von Auer (1999, S. 313) aufgeführte Methode ist die Analyse von Selbst- und Fremdreparaturen bezüglich der Sprachwahl, die die Orientierung der Teilnehmer auf die beiden Codes zeigt. 
interessieren und einzusetzen begannen. Die Kernthemen dieser Sozialwelt bestanden und bestehen darin, sich von ethnischer Diskriminierung im Einwanderungsland und von national-konservativen Aspekten ihrer Herkunftskultur zu emanzipieren.

Schon die Selbstbezeichnungen dieser neuen politischen Sozialwelt markieren den Unterschied zu den dominierenden Selbstorganisationen der Migranten aus der Türkei, die sich primär ethnisch definieren. Statt einer ethnischen Positionierung bringen die Namen der „emanzipatorischen Migranten“ eine verstärkte Orientierung zum Einwanderungsland Deutschland und die Bikulturalitiät ihrer Akteure zum Ausdruck:

- „Kauderzanca“ ist eine Zusammensetzung der beiden Wörter „Kauderwelsch“ und dessen türkischer Entsprechung „Tarzanca“. Die Wortbildung spielt auf die Zweisprachigkeit dieser jungen Migranten an und geht offensiv mit der ihnen unterstellten „doppelten Halbsprachigkeit“ um.

- Die Bezeichnung „Saz-Rock“ rekurriert ebenfalls auf die Bikulturalität der Akteure, indem „Saz“, ein traditionelles Zupfinstrument in der Türkei, mit „Rock“, der Bezeichnung für eine westliche Musikrichtung, verbunden wird.

- „köXüz“ ist eine Verfremdung des türkischen Worts „köksüz“, das auf Deutsch „wurzellos“ bedeutet. Mit dem Buchstaben „X“, der in der deutschen und englischen aber nicht in der türkischen Orthografie existiert, wird der Bezug zu ihrem Status als Migranten und zu der antirassistischen Bewegung der Schwarzen in den USA der 1960er Jahre hergestellt. Auf der anderen Seite drückt die Schreibweise die Distanz der zweiten Generation zur Herkunftskultur der Eltern aus.

- Im Gegensatz zu diesen Gruppen verzichten „Die Unmündigen“ in ihrer Selbstbezeichnung gänzlich auf einen Bezug zum Herkunftsland. Dadurch bringen sie - obwohl ihre Gründungsmitglieder ausschließlich Migranten aus der Türkei waren - ihre multiethnische Orientierung zum Ausdruck. Der Hauptgrund für ihre Namensgebung besteht in ihrer Orientierung zum Einwanderungsland, die provokativ zur Sprache gebracht wird, indem sie ihren Status als politisch unmündige Bürger in Deutschland fokussieren. In ähnlicher Weise verfährt das gegen Ende der 1990er Jahre gegründete Netzwerk „Kanak Attak“. Auch sie verzichten in ihrer Selbstbezeichnung auf einen Bezug zu den Herkunftsländern und fokussieren dagegen offensiv die Diskriminierung der Migranten seitens der Mehrheitsgesellschaft, indem sie deren negative, alltagsrassistische Bezeichnung „Kanak“ übernehmen und positiv als Bezeichnung für alle Migranten jenseits ihrer ethnischen Herkunft besetzen. 
Für meine soziolinguistische Untersuchung habe ich die Mannheimer Gruppe der „Unmündigen“ als Fallstudie für die soziale Welt der „emanzipatorischen Migranten" teilnehmend beobachtet und Interaktionen des Vereins auf Video und Tonband aufgezeichnet. Die Gründungsmitglieder der „Unmündigen“ waren ausschließlich Migranten der zweiten Generation aus der Türkei. Bis zum heutigen Tage stellen sie die Mehrheit der Gruppenmitglieder dar. Gleichzeitig entwickelte sich die Gruppe in den folgenden Jahren nach ihrer Konstitution immer mehr zu einem multiethnischen Verein, als sich ihr u.a. Migranten italienischer, spanischer, griechischer und bosnischer Herkunft anschlossen.

Zu Beginn waren die „Unmündigen“ hinsichtlich des beruflichen Hintergrunds der Mitglieder sehr heterogen zusammengestellt. Es beteiligten sich Sozialarbeiter, Bankangestellte, Gastronomen, Studenten, Arbeiter, KfzMechaniker, Arbeitslose und Künstler. Ab 1994 begann sich diese Zusammensetzung langsam aber stetig zu verändern. Bereits 1996 war die überwiegende Mehrheit der Mitglieder Studenten. Bis zum heutigen Tage hat sich diese Entwicklung nicht verändert, bis auf den Umstand, dass die damaligen Studenten ihr Studium beendet haben und mittlerweile zum Teil als Akademiker und zum Teil als Filmemacher, Sozialarbeiter, Journalisten, Grafiker etc. im Berufsleben stehen.

Das Geschlechterverhältnis hielt sich bei den „Unmündigen“ fast immer die Waage. In den Anfangsjahren gab es Phasen, in denen Frauen die Mehrheit der Mitglieder stellten. Gegenwärtig sind etwa 40\% der Mitglieder Frauen. Zum Zeitpunkt der Ethnografie waren die meisten Gruppenmitglieder zwischen 25 und 30 Jahre alt.

\section{Sprachwahl und -variation der untersuchten Gruppe}

Die sprachliche Orientierung und der Umgang mit sprachlichen Ressourcen sind wesentliche Aspekte, mit denen sich soziale Einheiten nicht nur aus der Außenperspektive unterscheiden lassen. Auch und vor allem aus der Perspektive der untersuchten Akteure dienen sie häufig dazu, relevante Andere zu kategorisieren und hinsichtlich der eigenen Welt ein- bzw. auszugrenzen. Dabei spielen in Bezug auf Sprachwahl- und Variationsaspekte zwei Fragen sowohl für die Akteure als auch für die soziolinguistische Analyse eine zentrale Rolle:

1) In welchem Maße verwenden die Akteure in der gruppeninternen Kommunikation die Herkunftssprache und die Sprache der Einwanderungsgesellschaft, also im vorliegenden Fall Türkisch und Deutsch?

2) Variieren sie in der gruppeninternen Kommunikation zwischen den Sprachen? Wenn ja, in welcher Dichte und bei welchen Interaktionsaktivitäten? 
3.1 Zwei Typen von Gruppeninteraktionen hinsichtlich sprachlicher Orientierung und Variation

Zur Analyse der Sprachorientierung und -variation der „Unmündigen“ wurden insgesamt 16 Treffen der Gruppe untersucht. Durchschnittlich haben diese Interaktionen eine Länge von etwa 100 Minuten. In einem ersten Analyseschritt wurden zunächst alle Treffen quantitativ erfasst, und zwar hinsichtlich der Frage, welche Akteure in der Interaktion wie viele Beiträge in Deutsch, Türkisch oder deutsch-türkisch gemischt formuliert haben.

Die quantitative Analyse der Gruppentreffen brachte zwei prototypische Verläufe von Interaktionen hervor. Die überwiegende Mehrheit der Treffen folgt einem Interaktionstyp A (siehe Tabelle 1), bei dem die Beteiligten fast ausschließlich Deutsch als Interaktionssprache verwenden.

\begin{tabular}{|l|r|r|r|r|r|c|}
\hline \multicolumn{7}{|c|}{ Interaktionstyp A } \\
\hline $\begin{array}{l}\text { Sprecher } \\
\text { Redebeiträge }\end{array}$ & BY & HE & ZA & CI & AD & $\begin{array}{c}\text { Redebeiträge } \\
\text { insg. }\end{array}$ \\
\hline türkische R. & - & - & - & 2 & - & 2 \\
\hline deutsche R. & 246 & 149 & 191 & 92 & 41 & 719 \\
\hline $\begin{array}{l}\text { deutsch-türkisch } \\
\text { gemischte R. }\end{array}$ & - & - & 1 & - & - & 1 \\
\hline Rest ${ }^{8}$ & 22 & 5 & 8 & 4 & 1 & 40 \\
\hline Redebeiträge insg. $^{\text {Regre }}$ & 268 & 154 & 200 & 98 & 42 & 762 \\
\hline
\end{tabular}

Tab. 1: Interaktionstyp A

Elf der untersuchten 16 Gruppentreffen entsprechen dem Interaktionstyp A. Wie aus der Tabelle 1 hervorgeht, sind die Teilnehmer bei diesen Interaktionen nahezu monolingual Deutsch orientiert. Auf jeden Fall ist Deutsch die unbestrittene Interaktionssprache. Nur drei von insgesamt 762 Redebeiträgen sind bei der in Tabelle 1 ausgewerteten Interaktion deutsch-türkisch gemischt oder nur Türkisch. In Abschnitt 3.2 wird ausgeführt, dass es sich bei diesen wenigen Fällen von Sprachalternationen um lokal bedeutungsvolles Code-Switching handelt. Aber bereits an dieser Stelle kann man festhalten, dass diese Sprachpraxis hinsichtlich des kommunikativen Stils der „emanzipatorischen Migranten“ sehr bedeutsam ist. Im Gegensatz zu anderen Migrantenmilieus, die in ihrer gruppen-

Die Kategorie „Rest“ enthält Sprecherbeiträge, die entweder aus nicht-sprachlichen Hörersignalen bestehen oder Äußerungen enthalten, die nicht verständlich sind. 
internen Kommunikation vielmehr die Herkunftssprache verwenden, drücken sie damit ihre uneingeschränkte Orientierung zum Einwanderungsland Deutschland aus.

Neben diesem bei den „Unmündigen“ dominanten Interaktionsverlauf gibt es aber auch einen zweiten Interaktionstyp, der sich durch vermehrte Sprachalternation auszeichnet. Wie in Tabelle 2 deutlich wird, nehmen bei diesem Interaktionstyp B die Wechsel von der dominanten Interaktionssprache Deutsch zu Türkisch deutlich zu.

\begin{tabular}{|l|r|r|r|r|r|c|}
\hline \multicolumn{7}{|c|}{ Interaktionstyp B } \\
\hline $\begin{array}{l}\text { Sprecher } \\
\text { Redebeiträge }\end{array}$ & YA & ME & AR & TU & AL & $\begin{array}{c}\text { Redebeiträge } \\
\text { insg. }\end{array}$ \\
\hline türkische R. & 5 & 12 & 1 & 7 & - & 25 \\
\hline deutsche R. & 53 & 32 & 29 & 24 & 14 & 152 \\
\hline $\begin{array}{l}\text { deutsch-türkisch } \\
\text { gemischte R. }\end{array}$ & 6 & 5 & 5 & 2 & 1 & 19 \\
\hline Rest & 11 & 3 & 6 & 3 & 3 & 26 \\
\hline Redebeiträge insg. & 75 & 52 & 41 & 36 & 18 & 222 \\
\hline
\end{tabular}

Tab. 2: Interaktionstyp B

Fünf der untersuchten 16 Treffen entsprechen dem Interaktionstyp B. Auch bei diesem Interaktionstyp ist Deutsch die dominante Interaktionssprache. Aber die Anzahl von türkischen und deutsch-türkisch gemischten Redebeiträgen ist deutlich mehr (etwa 20\% der Redebeiträge) als beim Interaktionstyp A. Wie später in Abschnitt 3.3 ausgeführt wird, handelt es sich bei diesen Sprachalternationen neben Code-Switching auch um Code-Mixing, d.h. um Sprachwechsel, die sequenziell nicht mehr bedeutungsvoll sind.

\subsection{Code-Switching beim Interaktionstyp A}

Aus den tabellarischen Darstellungen geht hervor, dass bei den „Unmündigen“ der Interaktionstyp A das häufigste Interaktionsmuster darstellt. Danach verwenden die Beteiligten fast die gesamte Interaktion über Deutsch. Nur an einigen wenigen Stellen alternieren sie sequenziell zwischen den Sprachen. Diese lokalen Sprachwechsel lösen in der Regel keine Interaktionspassagen aus, in denen dicht und über einen längeren Zeitraum zwischen den Sprachen alterniert wird.

Siehe z.B. in Keim/Cindark (2003) oder Zifonun/Cindark (2004). Und zur soziopolitischen Bedeutung der Sprachwahl bei der Konstitution der „Unmündigen“ siehe in Cindark (2010, S. 101-107). 
Im Folgenden werde ich vier Fallbeispiele für diese kleinräumigen Sprachalternationen analysieren, die für den kommunikativen Sozialstil der „Unmündigen“" charakteristisch sind. Wie die Fallbeispiele verdeutlichen werden, verwenden die Beteiligten Code-Switching in vielen verschiedenen Funktionen. Zu den häufigsten sozialen und interaktionalen Funktionen, die lokale Sprachalternationen bei den „Unmündigen“ erfüllen, zählen Sprachwechsel zur Markierung von Nebensequenzen, spielerischer und ernster Kritik/Ermahnung und Modalitätswechsel.

\section{Fallbeispiel 1: Markierung einer Nebensequenz}

Im ersten Fallbeispiel handelt es sich um eine Interaktionspassage, die sich vor Beginn des offiziellen Teils einer Gruppensitzung ereignet. Die Gruppentreffen der „Unmündigen“ verlaufen in der Regel so, dass sich die Mitglieder zunächst im Café der Kultureinrichtung zusammenfinden, in der sie ihre Treffen abhalten. Wenn alle Mitglieder eingetroffen sind, zieht sich die Gruppe dann für ihre Sitzung in einen der Clubräume zurück. Die typischen Interaktionsaktivitäten im Café bestehen darin, dass sich die Mitglieder gegenseitig begrüßen, kurze Alltagsgespräche führen, Getränke bestellen und in unfokussierter Weise ihre Post durchgehen. In der folgenden Interaktionspassage sitzen mehrere Mitglieder am Tisch, während NI kommentierend diverse Postzusendungen an die Gruppe durchgeht. KR ist gerade angekommen und hat sich neben NI gesetzt.

\section{Transkript: ne yapiyon $\mathrm{KR}$}

\begin{tabular}{|c|c|c|}
\hline 01 & $\begin{array}{l}\mathrm{NI}: \\
\mathrm{K}\end{array}$ & 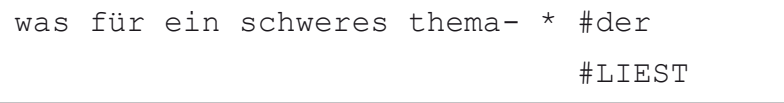 \\
\hline 02 & $\begin{array}{l}\mathrm{NI}: \\
\mathrm{K}\end{array}$ & $\begin{array}{l}\text { erwerb sozialer und kommunikativer } \\
\text { EINE EINLADUNG VOR }\end{array}$ \\
\hline 03 & $\mathrm{NI}:$ & schlüsselkompe/ kompetenzen \\
\hline 04 & NI : & in der jugendarbeit $\downarrow$ und re"ssorts für| \\
\hline 05 & NI : & andere lebensbereiche $\downarrow$ * wie zum beispiel \\
\hline 06 & $\begin{array}{l}\text { NI : } \\
\ddot{U}\end{array}$ & 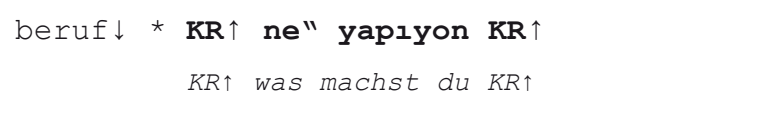 \\
\hline 07 & $\begin{array}{l}\mathrm{KR}: \\
\ddot{\mathrm{U}}\end{array}$ & $\begin{array}{l}\text { ne yapayım } \\
\text { was soll ich }\end{array}$ \\
\hline
\end{tabular}




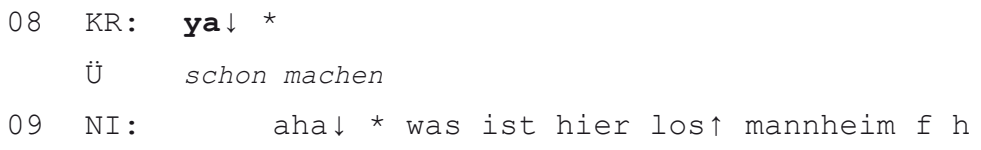

$\mathrm{Zu}$ Beginn des Ausschnitts geht NI die Briefsendungen an die Gruppe durch. Von Zeile 01 bis 06 kommentiert er zunächst den Untertitel eines Seminars, zu dem die „Unmündigen“ eingeladen werden: was für ein schweres thema- (Z. 01). Anschließend liest er den Untertitel vor: der erwerb sozialer und kommunikativer schlüsselkompe/ kompetenzen in der jugendarbeit $\downarrow$ und re"ssorts für andere lebensbereiche $\downarrow$ * wie zum beispiel beruf $\downarrow$ (Z. 01-06). Nachdem NI den Titel des Seminars vorgelesen hat, wendet er sich in Zeile $06 \mathrm{KR}$ zu und begrüßt ihn mit einer türkischen Routineformel: $K R \uparrow n e$ "yapyon $K R \uparrow(\mathrm{KR} \uparrow$ was macht du KR $\uparrow$ ). KR reagiert auf NIs Begrüßung ebenso auf Türkisch

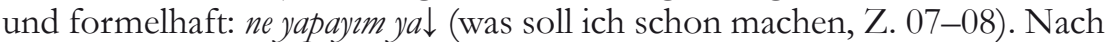
dieser Sequenz aus formelhafter Begrüßung und Erwiderung kehrt NI wieder zu seiner vorherigen Aktivität zurück, diverse Postzusendungen an die Gruppe durchzugehen. Dabei wechselt er ebenso zu seiner vorherigen Interaktionssprache Deutsch: aha ${ }^{*}$ was ist hier los $\uparrow *$ mannheim $f b^{*}(\mathrm{Z} .11-$ 12). Interaktionsstrukturell markiert die Sprachalternation von Deutsch zu Türkisch zwischen NI und KR an dieser Stelle eine Nebensequenz aus zwei kurzen Routineformeln zur Begrüßung. In der Gruppeninteraktion der „Unmündigen“ kommen solche sequenziell kleinräumigen Sprachalternationen zur Markierung von vielfältigen Einschüben vor. Im analysierten Fall handelt es sich um eine Nebensequenz, in anderen Fällen zum Beispiel um metapragmatische Kommentare.

\section{Fallbeispiel 2: Markierung einer spielerischen Kritik/Ermahnung}

Das Deutsch der „Unmündigen“ ist bis auf das von einem Mitglied (BÜ), der erst mit sechzehn Jahren nach Deutschland kam, weitestgehend standardnah und grammatisch unauffällig. Dass die Dominanz des Deutschen einerseits die individuellen Kompetenzen reflektiert, aber andererseits für die sozialweltliche Präferenz der „emanzipatorischen Migranten“ steht, lässt sich insbesondere am sprachlichen Verhalten von eben diesem Mitglied beobachten. Obwohl BÜs Performanz im Deutschen immer wieder Prozessierungsprobleme zeigt, stellen diese für ihn in der Regel keinen Grund dar, in den bilingualen Modus zu wechseln. Er bleibt im monolingual deutschen Modus, gleichwohl fehlerhaft, und reagiert auf Fremdreparaturen, wie im folgenden Transkriptausschnitt deutlich wird, ausgesprochen positiv. In der Interaktion wird BÜ von DS korrigiert. Auf diese potenziell gesichtsbedrohende Aktivität reagieren zwei Anwesende (MA und TA), in dem sie zum Teil oder ganz ins Türkische wechseln. Das Besondere an die- 
sem Fallbeispiel ist, dass TA und MA, die hier ins Türkische wechseln, italienischer Herkunft sind, was in der soziolinguistischen Forschung als „language crossing" (Rampton 1995) bezeichnet wird.

\section{Transkript: eine foyer}

\begin{tabular}{|c|c|c|}
\hline 02 & BÜ : & ku"nstverein ist * die nächsten zwei jahren \\
\hline 03 & BÜ : & ausgebucht $\uparrow * \star$ und $i /$ in kunst|verein| \\
\hline 04 & DS : & | LACHT | \\
\hline 05 & $\mathrm{LE}:$ & | LACHT | \\
\hline \multirow[t]{2}{*}{06} & BÜ : & | LACHT \#wa"rte mal| wa"rte mal $\downarrow$ \# * \\
\hline & $\mathrm{K}$ & \#LEICHT LACHEND \\
\hline 07 & DS : & $\mid=\mathrm{LACHT}$ \\
\hline 08 & BÜ : & in kunstverein $\uparrow$ soll eine fo"yer geben $\uparrow$ \\
\hline 09 & DS : & 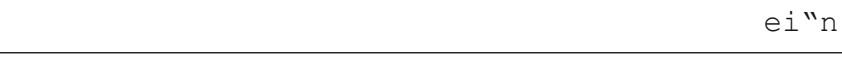 \\
\hline 10 & DS : & $\star \leftarrow$ foyer $\rightarrow *$ \\
\hline 11 & BÜ : & ei"n foyer $\downarrow$ * der oder das \\
\hline 12 & BÜ : & foyer $\uparrow \quad \mid>$ das foyer $\downarrow<1$ \\
\hline 13 & DS : & da"s $\mid \leftarrow$ foyer $\downarrow \rightarrow$ \\
\hline 14 & $\mathrm{MA}:$ & andere \\
\hline \multirow[t]{2}{*}{15} & $\mathrm{MA}:$ & | bau|stelle |lan $\downarrow \mid$ \\
\hline & $\ddot{\mathrm{U}}$ & Kerl \\
\hline 16 & BÜ : & |also $\mid$ \\
\hline \multirow[t]{2}{*}{17} & TA: & |su"s | lan $\downarrow$ \\
\hline & $\ddot{\mathrm{U}}$ & sei still, Kerl \\
\hline
\end{tabular}

Mit „language crossing“ werden Sprachalternationen von Sprechern beschrieben, die nicht Mitglieder einer Sprachgemeinschaft sind, deren Sprache sie sprechen oder zumindest Versatzstücke oder Formeln dieser Sprache beherrschen und in der Interaktion einsetzen. ${ }^{10}$ Speziell in multiethnischen

$10 \quad$ Siehe dazu neben Rampton (1995) vor allem die Studie von Dirim/Auer (2004), die Fälle von „language crossing“ in Deutschland, genauer von Jugendlichen in Hamburg, die nicht-türkischer Herkunft sind, aber in verbalen Interaktionen teils oder ganz Türkisch sprechen. 
Gruppen ist diese Form des Code-Switchings ein wesentliches Merkmal der multilingualen Praxis. Bei den „Unmündigen“ ist diesbezüglich das Kommunikationsverhalten von TA und MA von Interesse, die beide italienischer Herkunft sind. ${ }^{11}$ Im Transkriptausschnitt reagieren MA (Z. 14-15) und TA (Z. 17) auf die Fremdkorrektur DSs (Z. 09-10), die sie für BÜ, dem Korrigierten, als gesichtsbedrohende Aktivität interpretieren. Sie reagieren auf den interaktiv ,heiklen' Vorfall, kontextualisieren aber mit dem Sprachwechsel eine spielerische Modalität.

MA verwendet eine stereotype Formel aus dem „Gastarbeiterdeutsch“, andere baustelle (vgl. Cassisi 2008), zusammen mit der derben türkischen Anrede lan $\downarrow$ (Kerl, Z. 14-15). Damit referiert er spielerisch auf die situative Inadäquatheit der Äußerung DSs im Sinne von „deine Korrektur BÜs ist fehl am Platz". TA reagiert mit der in Türkisch formulierten, imperativischen Aufforderung su"s lan $\downarrow$ (sei still Kerl, Z. 17). ${ }^{12}$ Solche Wechsel zu stilisierten Formen des „Gastarbeiterdeutsch“ oder Fälle von ,language crossing “ sind in der Ingroup-Kommunikation der „Unmündigen“ wichtige Variationspraktiken zwischen den türkisch- und anderssprachigen Mitgliedern, mit denen sie lokal eine spielerische Modalität kontextualisieren und global interethnische Gemeinsamkeit anzeigen.

\section{Fallbeispiel 3: Markierung einer ernsten Kritik/Ermahnung}

In den bisherigen Fallbeispielen wurde bereits deutlich, dass aufgrund der Sprachorientierung der „Unmündigen“, die sich weitestgehend durch Deutsch als Gruppensprache auszeichnet, einzelne sequenzielle Sprachalternationen wichtige lokale Interaktionsfunktionen erfüllen. Das folgende Beispiel ist ein sehr interessanter Beleg dafür, welche immense Bedeutung sogar eine minimale lokale Sprachalternation, in dem Fall bestehend aus nur einem türkischen Wort in einer ansonsten monolingual deutschen Interaktionspassage, entwickeln kann.

Zum Kontext: Der Transkriptausschnitt stammt aus einer Sitzung, in der die Beteiligten besonders ausgelassen und scherzhaft interagieren. Sie kommen kaum dazu, die anfallenden Tagesordnungspunkte zu besprechen. $\mathrm{Zu}$ Beginn der Sitzung hatte ein Mitglied (FU) angekündigt, dass sie früher

${ }_{11}$ Beide sind in einem Mannheimer Stadtteil (Schönau) geboren und aufgewachsen, der von vielen italienischen und türkischen Migranten bewohnt ist. In Kontakt mit türkischen Kindern und Jugendlichen hat insbesondere TA viele türkische Ausdrücke und formelhafte Wendungen erworben. Wie er mir im ethnografischen Interview mitteilte, könne seine (ebenfalls italienischstämmige) Schwester, deren beste Freundin eine Türkin sei, sogar flieBend Türkisch.

12 Nach Rampton (1995) können Fälle von „language crossing“ verschiedene Interaktionsfunktionen erfüllen. So kommen sie u.a. in Kontexten von ritualisierten Beschimpfungen oder bei Verletzungen der Interaktionsregeln vor (ebd., S. 281), wozu auch die Äußerungen von MA und TA gerechnet werden können. 
gehen muss und deshalb darum gebeten, zügig die Tagesordnungspunkte zu bearbeiten. In der folgenden Passage ist jedoch ihre interaktive Beteiligungsweise, Füße auf dem Tisch und mit einer Nagelfeile ihre Nägel säubernd, relativ leger bis ablenkend. Diese Haltung, konkret ihr Nägelfeilen, löst eine scherzhafte Äußerung eines Anwesenden (RT) aus, worauf ein anderer Beteiligter (IP) mit einem türkischen Ordnungsruf reagiert.

\section{Transkript 17: lütfen}

\begin{tabular}{|c|c|c|}
\hline 01 & IP : & $\# \star 2 * \# \quad$ weiter $\downarrow$ \\
\hline 02 & SO: & was war als nächstes $\uparrow$ \\
\hline \multirow[t]{2}{*}{03} & $\mathrm{RT}:$ & I LACHT \\
\hline & $K \&$ & \#ALL\#E LACHEN \\
\hline 04 & IP : & wegen | diese presse*sachen |äh wo:- | \\
\hline 05 & $\mathrm{RT}:$ & $\mid>F U \uparrow$ gibst $\mid d u$ \\
\hline 06 & $\mathrm{RT}:$ & mir dann wenn du fertig bist weil ich muss \\
\hline 07 & $\mathrm{RT}:$ & auch fertig |machen ||$(\ldots)<1$ \\
\hline 08 & SO: & lja- \\
\hline 09 & IP : & FU $\downarrow \mid$ lütfen $\downarrow$ \\
\hline & $\ddot{\mathrm{U}}$ & bitte $\downarrow$ \\
\hline
\end{tabular}

10 So: |pre"ssesachen $\downarrow \mid$

$11 \mathrm{FU}: \mid$ ATMET EIN $\mid<I P \downarrow$ moment mal $\downarrow$ * was

12 FU: so"lln das jetzt $\downarrow>\mid$ e"r spricht| mich

13 IP: |also ich finde-|

14 FU: hier an und du sagst $\rightarrow$ FU lütfen $\downarrow$ kannst du

15 FU: mir erklären was das jetzt $\leftarrow$ bedeutet $\uparrow$

16 IP: $\quad \leftarrow \mathrm{FU} \rightarrow$

17 IP: |kuck=ma| ich find das- äh also e"rstens du

18 FU: |also

19 IP: sagst dass wir ernst nehmen- und dass- ich

20 IP: |trenn des- | |okay aber-|

21 FU: |<ich nehm des| ernst $\downarrow>\mid$ stö"rt das | 


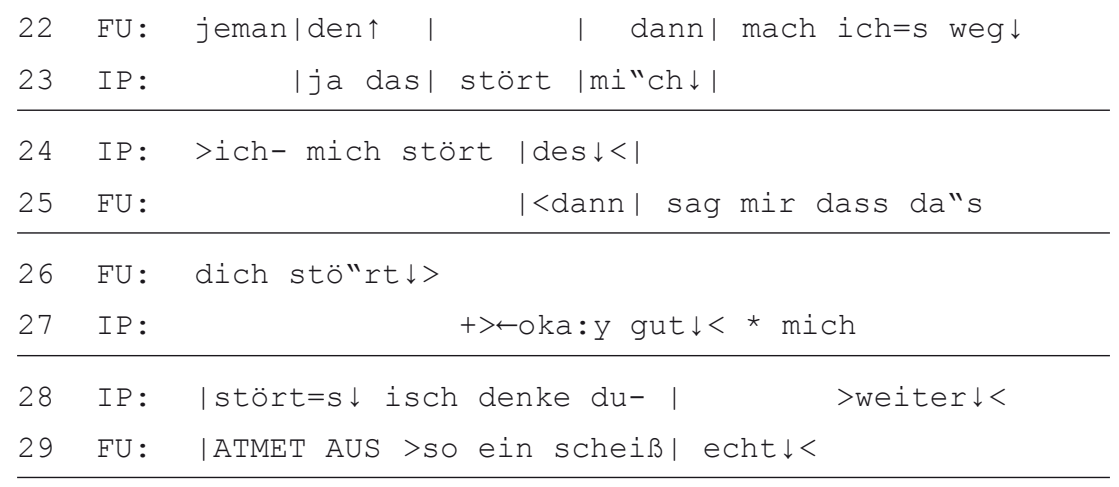

Der Trankriptausschnitt beginnt nach einer scherzhaften Interaktionspassage, als die Anwesenden noch herzhaft lachen. In Zeile 01 fordert IP die Anwesenden formelhaft auf, sich wieder auf die Tagesordnung zu konzentrieren: weiter $\downarrow$. Darauf reagiert SO mit einer Rückfrage: was war als nächstes $\uparrow$ (Z. 02). Parallel zu IPs folgendem Redebeitrag, in dem er den Tagesordnungspunkt „Pressesachen“ thematisiert (Z. 01-04), initiiert RT eine Nebenaktivität: Zunächst leicht lachend (Z. 03-05) richtet er eine leise, als off-record markierte Frage an FU: $>F U \downarrow$ gibst du mir dann wenn du fertig bist weil ich muss auch fertig machen < (Z. 05-07). RTs Frage zielt auf die Nagelfeile, mit der sich FU seit geraumer Zeit die Fingernägel säubert. Durch sein leichtes Lachen vor seinem Redebeitrag kontextualisiert RT seine Äußerung als eine scherzhafte Aktivität, wodurch die von IP und SO gerade etablierte ernsthafte Modalität erneut in Gefahr gerät, verdrängt zu werden. Im Folgenden reagieren SO und IP in unterschiedlicher Weise auf diese ,Interaktionsgefahr': SO ignoriert die Äußerung RTs und versucht als legitimierter nächster Sprecher (er ist der Verantwortliche für die Pressearbeit) mit der Tagesordnung fortzufahren: ja- pre"ssesachen $\downarrow$ (Z. 08-10). IP reagiert auf die Nebenaktivität

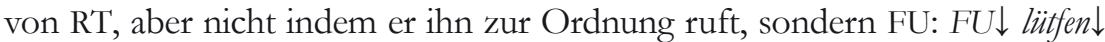
(bitte, Z. 09). Mit dieser höflichen aber gleichsam nachdrücklichen Ermahnung spielt er auf die ablenkende Wirkung ihres Nägelfeilens an.

IPs „Bitte“ ist ein Ordnungsruf, der in doppelter Weise an FU adressiert ist, und zwar durch Namensnennung und durch Sprachwechsel ins Türkische. Durch den Sprachwechsel ist RT, der formal-verbal als Auslöser der störenden Sequenz betrachtet werden kann, als Adressat ausgeschlossen; er ist nicht türkischer Herkunft und versteht daher nur bedingt Türkisch. Dieser doppelt adressierte Ordnungsruf löst bei der kritisierten FU Unverständnis und Ärger aus. Sie unterbricht den aktuellen Sprecher (SO) und wendet sich - in auffälliger Analogie zu IPs Formulierung - namentlich an den Kritiker und weist seine Kritik zurück: $\langle I P \downarrow$ moment mal $\downarrow$ * was so "Iln das jetzt $\downarrow>$ (Z. 11-12). Die Äußerung FUs weist folgende argumentative Struktur auf: 
- Zurückweisung der Kritik als ungerechtfertigt: $<I P \downarrow$ moment mal $\downarrow$ * was so"lln das jet: $\downarrow>$ (Z. 11-12).

- Begründung der Zurückweisung, die sequenziell fundiert wird, indem RTs Aktivität als Störquelle fokussiert wird: $e$ "r spricht mich bier an und $d u$ sagst $\rightarrow$ FU lütfen $\leftarrow$ (Z. 12-14).

- Aufforderung zur Explikation der Kritik: kannst du mir erklären was das jetzt $\leftarrow$ bedeutet $\uparrow($ Z. 14-15).

Parallel zu FUs Begründung in Zeile 12 startet IP einen ersten Versuch, seinen Ordnungsruf zu rechtfertigen also ich finde- (Z. 13). Er bricht aber seine Formulierung ab und wartet, bis er von FU eindeutig als nächster Sprecher adressiert wird. Im zweiten Ansatz versucht IP durch vorgeschaltete Namensnennung und Aufmerksamkeitsformel zunächst die Kooperativität FUs zu sichern: $\leftarrow F U \rightarrow$ kuck $=m a$ (Z. 16-17), und beginnt dann in Form einer argumentativen Aufzählung die Gründe für seinen Ordnungsruf zu explizieren: ich find das- äh also e "rstens du sagst dass wir ernst nehmen und dass-ich trenn des- (Z. 17-20). Er zitiert FU und erinnert an die grundlegend unterstellte Orientierung der Mitglieder, die Besprechung der organisatorischen Aufgaben nicht zu vernachlässigen. Diese Orientierung erfordert die Anstrengung aller, zwischen ernster und spielerischer Modalität zu trennen. FU unterbricht ihn und reklamiert ihrerseits, die Sitzung ernst zu nehmen: <ich

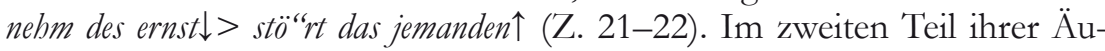
Berung referiert sie auf ihre vorherige Aktivität „Nägel feilen“, die sie als Auslöser für IPs Ordnungsruf betrachtet. Sie adressiert mit ihrer Frage nicht nur IP, sondern alle Anwesenden, um auch deren Situationsdefinition einzubeziehen.

IP und FU haben sich zu diesem Zeitpunkt als Streitparteien warmgelaufen, sie wenden beide forcierende Verfahren an und verhindern die Einmischung Dritter. ${ }^{13}$ IP lässt auch jetzt keine Reaktion der anderen zu und bestätigt, noch überlappend mit FUs Frage, das von ihr unterstellte Motiv für seinen Ordnungsruf: ja das stört mi “ $c b \downarrow$ (Z. 23). FU liefert eine schnelle Ausgleichshandlung: ${ }^{14}$ dann mach ich $=s$ weg $\downarrow$ (Z. 22) und zeigt damit ihre prinzipielle Kooperationsbereitschaft und Kritikfähigkeit an. Auf diese Ausgleichshandlung stuft IP seinen Vorwurf zurück und reduziert mit dem leiser und zurückgenommen gesprochenen: >ich- mich stört des $\downarrow<$ (Z. 24) dessen Geltungsanspruch.

\footnotetext{
Zu Forcieren als gesprächsrhetorisches Verfahren siehe Kallmeyer/Schmitt (1996).
}

14 Goffman (1978, S. 25 ff.) beschreibt das (rituelle) Schema von Ausgleichshandlungen, das aus den folgenden Schritten besteht: a) „Herausforderung“: Ein interaktiver Vorgang wird von einem Beteiligten als Angriff, Beleidigung etc. verstanden; b) „Angebot“: Der Missetäter macht ein Angebot zur Wiedergutmachung (er entschuldigt sich, verspricht Besserung u.Ä.); und c) „Angebotsannahme“: Die ,geschädigte“ Person nimmt das Wiedergutmachungsangebot an. 
Jetzt reagiert FU mit einer erneuten Offensive, die deutlich macht, dass für sie der zentrale Punkt des Konflikts noch offen ist: <dann sag mir dass da"s dich stö" $r t \downarrow>$ (Z. 25-26). Nicht die Kritik an sich betrachtet sie als problematisch, sondern zwei Aspekte des Ordnungsrufes:

- seine inhaltliche Unbestimmtheit: Er referiert nicht auf die inkriminierte Handlung und die Kritisierte kann den Kritikpunkt nur erahnen; und

- seine sequenzielle Platzierung: Der Ordnungsruf folgt auf eine spielerische Äußerung von RT an FU, weshalb für die Störung eigentlich RT und nicht FU verantwortlich ist; da sich IP jedoch durch FUs Nägelfeilen gestört fühlt, ist der Zeitpunkt, ihr das zu sagen, ungünstig gewählt, die Platzierung des Ordnungsrufes an sie ,heikel'.

Die sequenzielle Anbindung des Ordnungsrufes in Verbindung mit dem Sprachwechsel und der inhaltlichen Vagheit machen seinen problematischen Charakter aus. FU erreicht durch ihre diskursiv-kooperative Art das Einsehen von IP: $+>\leftarrow$ oka:y gut $\downarrow$ * mich stört=s $\downarrow$ isch denke du- (Z. 27-28). Mit einem deutlich hörbaren Ausatmen und dem leiser gesprochenen Kommentar: ATMET AUS > so ein scheiß echt $\downarrow<($ Z. 29) signalisiert FU das Ende der Konfliktbearbeitung, und IP ratifiziert den Abschluss, indem er mit der Kurzformel $>$ weiter $\downarrow<$ (Z. 28) zur Besprechung der Tagesordnungspunkte überleitet.

Bei den „Unmündigen“ kontextualisieren sequenziell kleinräumige Sprachalternationen in den meisten Fällen, wenn sie Aspekte der Modalität betreffen, einen Wechsel von der ernsten zur spielerischen Modalität. Das hier analysierte Beispiel zeigt jedoch, dass es auch Belege für die umgekehrte Richtung gibt. Auffällig an diesem Fallbeispiel ist, dass die beiden Streitparteien FU und IP, die beide türkischer Herkunft sind, bis auf das türkische Wort „lütfen“ (Bitte) zu Beginn, das die ganze Sequenz auslöste, während ihrer gesamten Auseinandersetzung kein zweites Mal vom Deutschen ins Türkische wechseln. Das unterstreicht ihre starke sozialweltliche Orientierung auf Deutsch als präferierte Gruppensprache.

\section{Fallbeispiel 4: Markierung eines Modalitätswechsels}

Auch mit dem nächsten und letzten Beispiel zu sequenziell kleinräumiger Sprachalternation liegt ein Fall vor, bei dem die Beteiligten Aspekte der Interaktionsmodalität und des Faceschutzes behandeln. Zum Kontext: Im präsentierten Ausschnitt besprechen die Beteiligten, bei welchem Mitglied eine Adressenliste abgeblieben sein könnte, die die Gruppe dringend braucht. Alle vermuten, dass BI, ein anwesendes Gruppenmitglied, die Liste zuletzt hatte. Als ein Beteiligter erneut allgemein fragt, wer wohl die Liste hat, ereignet sich Folgendes: 
Transkript: die adressenliste

\begin{tabular}{|c|c|c|}
\hline 01 & $\mathrm{WE}:$ & wer hat was $\uparrow \quad$ ds=mein ich $\downarrow$ \\
\hline 02 & AM: & die adressenliste $\downarrow$ \\
\hline 03 & $\mathrm{WE}:$ & $j a: \uparrow *$ \\
\hline 04 & $B I:$ & ich hab eine zuhause $\downarrow$ \\
\hline \multirow[t]{2}{*}{05} & OL: & $\operatorname{bak} \uparrow \star$ \\
\hline & $\ddot{U}$ & schau $\uparrow *$ \\
\hline \multirow[t]{2}{*}{06} & OL : & zuhausede olduğunda bu"rda olduğunu \\
\hline & $\ddot{\mathrm{U}}$ & wenn du zuhause bist sagst du dass sie hier \\
\hline \multirow[t]{2}{*}{07} & OL: & söylüyosun * burda olduğunda zuhau"sede \\
\hline & $\ddot{U}$ & ist * wenn du hier bist sagst du dass sie zuhause \\
\hline \multirow[t]{3}{*}{08} & OL: & $>0 l d u g ̆ u n u$ | söylüyosun $\downarrow$ \#olur mu $\uparrow<\# \mid$ \\
\hline & $\ddot{\mathrm{U}}$ & ist $\downarrow$ geht das $\uparrow$ \\
\hline & $\mathrm{K}$ & \#SCHMUNZEL\#ND \\
\hline 09 & AM: & | LACHT \\
\hline \multirow[t]{2}{*}{10} & $\mathrm{AM}:$ & \#flexibel- * die liste $\downarrow \#$ \\
\hline & K & \#LACHEND \\
\hline
\end{tabular}

Vor dem Beginn des Transkriptausschnitts hatte AM nach einer Adressenliste gefragt, die die „Unmündigen“ seit einiger Zeit vermissen. Parallel zu seiner Frage sprach jemand gleichzeitig. Aufgrund dieser Simultanpassage stellt WE in Zeile 01 an AM die Frage: wer hat was $\uparrow$. AM antwortet mit die adre"ssenliste $\downarrow$ (Z. 02), worauf WE die Relevanz des Themas bzw. der Frage

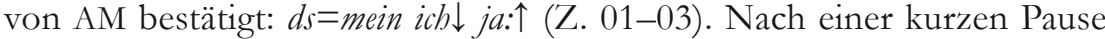
reagiert $\mathrm{BI}$, von der alle annehmen, dass sie die einzig vorhandene Liste hatte, zweideutig: ich hab eine zuhause $\downarrow$ (Z. 04). Ihre Äußerung ist ausweichend, da sie durch die Verwendung des unbestimmten Artikels unterstellt, dass es mehrere Exemplare der Liste geben würde. Im folgenden Redebeitrag nimmt OL in Zeile 05-08 nicht auf diesen Punkt Bezug, sondern generell auf den ausweichenden Charakter von BIs Verhalten: bak $\uparrow *$ zuhausede olduğunda bu"rda olduğunu söylïyosun burda olduğunda zuhau"sede >olduğunu söllüyosun $\downarrow$ olur mu $\uparrow<$ (schau, wenn du zuhause bist, sagst du, dass sie hier ist, wenn du hier bist, sagst du, dass sie zuhause ist, geht das). Inhaltlich deckt OL das ausweichende Verhalten BIs auf. Aber auf der sprachlichen Ebene kontextualisiert sie mit dem Wechsel in den deutsch-türkischen Mischcode ${ }^{15}$

15 Der Begriff Mischcode wird hier aufgrund der Einbettung des deutschen Elements ,zuhause“ in den türkischen Matrixsatz verwendet. Eine alternative Interpretation wäre, hier nicht vom 
eine spielerische Modalität, die durch die leicht lachende Artikulation gegen Ende der Äußerung (Z. 08) verstärkt wird, und stuft so die für BI heikle Sequenz etwas herab. ${ }^{16}$ Auf den Modalitätswechsel reagiert AM, parallel zu OLs Äußerung, mit Lachen und dann mit einem ironischen Kommentar, in dem er den Fokus von SE auf die Liste lenkt: is flexibel- * die liste $\downarrow$ (Z. 09-10).

In diesem Abschnitt habe ich vier Fallbeispiele für sequenziell kleinräumige Sprachalternationen untersucht. Diese Fälle stehen für die häufigsten Formen von lokalen Sprachalternationen, wie sie bei den „Unmündigen“ vorkommen. Mit sequenziell kleinräumigen Alternationen ins Türkische kontextualisieren die Beteiligten u.a. Nebensequenzen, spielerische/ernste Kritik und Modalitätswechsel verbunden mit Aspekten des Faceschutzes. Andere Funktionen von Sprachalternationen, wie sie bei den „Unmündigen" zu beobachten sind und die hier nicht untersucht wurden, sind z.B. Sprachwechsel zur Markierung von Kommentaren und Medienzitaten.

\subsection{Code-Switching und Code-Mixing beim Interaktionstyp B}

Neben den soeben analysierten Fällen von Code-Switching, die in der gruppeninternen Kommunikation der „Unmündigen“ die Mehrheit der Sprachwechselphänomene ausmachen, kommen in den Gruppentreffen auch immer wieder Interaktionspassagen vor, in denen die Beteiligten über eine längere Zeitspanne (bis zu 20 Minuten) zwischen Deutsch und Türkisch alternieren. Diese Fälle, die ich als sequenziell großräumige Sprachalternationen bezeichnen möchte, sind für den Interaktionstyp B charakteristisch, der sich im Vergleich zu Interaktionstyp A durch eine Zunahme von Sprachwechseln auszeichnet. Ein Charakteristikum dieser sequenziell großräumigen Sprachalternationen ist, wie man im folgenden Fallbeispiel sehen wird, dass darin Fälle von Code-Switching und Code-Mixing nebeneinander zu beobachten sind.

Zum Kontext der folgenden Interaktion: Nachdem alle Mitglieder im Café des Kulturhauses eingetroffen sind, beschließen sie für die Dauer der Sitzung, sich in einen der Clubräume zurückzuziehen. ME, YA und TU

Wechsel in den Mischcode, sondern einem Wechsel ins Türkische zu sprechen. Das deutsche Element ,zuhause“ würde dann als eine Insertion betrachtet, die zur textuellen Kohärenzherstellung (vgl. Auer 1998, S. 7) in der Ursprungssprache Deutsch zitiert und nicht auf Türkisch ,übersetzt‘ wird. Zur Unterscheidung von „,matrix language“ und „embedded lan-

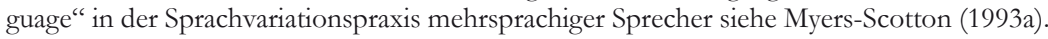

16 Der Modalitätswechsel wird hier doppelt, mit anderen Worten redundant, kontextualisiert: a) durch Codewechsel und b) durch das Schmunzeln. Zur Redundanz von Kontextualisierungshinweisen schreibt Auer (1984, S. 18): „Research on contextualization has shown that transitions between activity types are often marked on more than one level, thereby securing understanding even in cases where attention is given to some signalling channels only, or where participants do not necessarily share knowledge about contextualization conventions." 
gehen vor. Der Transkriptausschnitt beginnt in dem Moment, als die drei in den Clubraum eintreten. Bis zu diesem Zeitpunkt führten die Beteiligten ein scherzhaftes Gespräch über die Beziehung von ME zu einem anderen Mitglied der „Unmündigen“, das mit der Äußerung von ME in Zeile 01 abgeschlossen wird. Mit MEs Äußerung in Zeile 02 (YA bitte nicht aufmachen $\downarrow$ ) beginnt ein neuer thematischer Strang; sie bittet YA, der gerade das Fenster im Clubraum aufmachen will, weil die Luft im Zimmer stickig ist, das nicht zu tun. Dieser Abschnitt wird ab Zeile 10 von einem neuen Thema abgelöst, als ME beginnt, von ihrer aktuellen Gehaltserhöhung zu erzählen; das bleibt bis zum Ende des Transkriptausschnitts Thema des Gesprächs zwischen ihr und YA.

Transkript: zweifeln yapıyormus

\begin{tabular}{|c|c|c|}
\hline 01 & $\begin{array}{l}\mathrm{ME}: \\
\ddot{U}\end{array}$ & $\begin{array}{l}\leftarrow \text { BO benim platonik aşkım ama hiç kimse } \\
\leftarrow B O \text { ist meine platonische Liebe aber das weiß }\end{array}$ \\
\hline 02 & $\begin{array}{l}\mathrm{ME}: \\
\ddot{U}\end{array}$ & $\begin{array}{l}\text { bilmiyo bunu } \downarrow \rightarrow \text { şimdilik- * YA bitte nicht } \\
\text { niemand } \rightarrow \text { bis jetzt- }\end{array}$ \\
\hline $\begin{array}{l}03 \\
04\end{array}$ & $\begin{array}{l}\mathrm{ME}: \\
\mathrm{YA}: \\
\ddot{U}\end{array}$ & 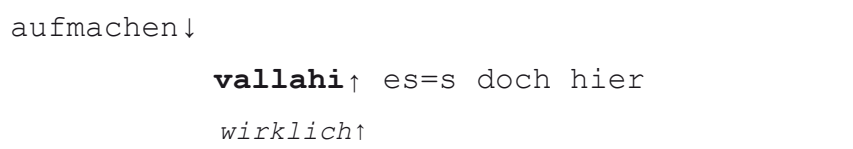 \\
\hline 05 & $\begin{array}{l}\text { YA : } \\
\ddot{U}\end{array}$ & $\begin{array}{ll}\mid(\text { stickig) } \mid & \text { dur } \\
& \text { warte }\end{array}$ \\
\hline $\begin{array}{l}06 \\
07\end{array}$ & $\begin{array}{l}\mathrm{TU}: \\
\mathrm{ME}:\end{array}$ & $\begin{array}{l}|(\ldots) \quad| \text { es war so angenehm } \downarrow \text { so warm } \downarrow\end{array}$ \\
\hline 08 & $\begin{array}{l}\text { YA : } \\
\ddot{U}\end{array}$ & $\begin{array}{ll}\text { |biraz- } & \text { temiz hava } \mid \text { (girsin) } \\
\text { etwas- } & \text { frische Luft (rein lassen) }\end{array}$ \\
\hline 09 & $\begin{array}{l}\mathrm{TU}: \\
\ddot{\mathrm{U}}\end{array}$ & $\begin{array}{l}\mid \text { biraz| çıksın ya } \downarrow \\
\text { etwas raus lassen, Mensch } \downarrow\end{array}$ \\
\hline 10 & $\begin{array}{l}\mathrm{ME}: \\
\ddot{\mathrm{U}}\end{array}$ & $\begin{aligned} \mid & \leftarrow i y i \mid \\
& \leftarrow \text { gut }\end{aligned}$ \\
\hline 11 & $\begin{array}{l}\mathrm{ME}: \\
\ddot{U}\end{array}$ & $\begin{array}{l}\text { hadi çıksın } \downarrow \rightarrow \star 1,5 *<\text { hey } \uparrow>\text { heute war ich } \\
\text { dann soll sie ("die schlechte Luft", m.A.) raus } \downarrow\end{array}$ \\
\hline $\begin{array}{l}12 \\
13\end{array}$ & $\begin{array}{l}\mathrm{ME}: \\
\mathrm{YA}:\end{array}$ & wieder in frankfurt den ganzen tag ne $\begin{array}{r}\text { } \\
\text { mh } \downarrow\end{array}$ \\
\hline
\end{tabular}


$14 \mathrm{ME}$ : ja: orda bizim bereichsleitungu görüyorum $\uparrow$

$\ddot{U} \quad$ dort sehe ich unsere Bereichsleitung $\uparrow$ so

15 ME: böyle çok önemli $\downarrow$ yukarlardan bi adam $\downarrow$ (er)

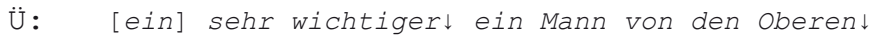

$16 \mathrm{ME}$ : hat gesagt-kommen sie kurz in |mein |

17 YA: |echt $\uparrow \mid$

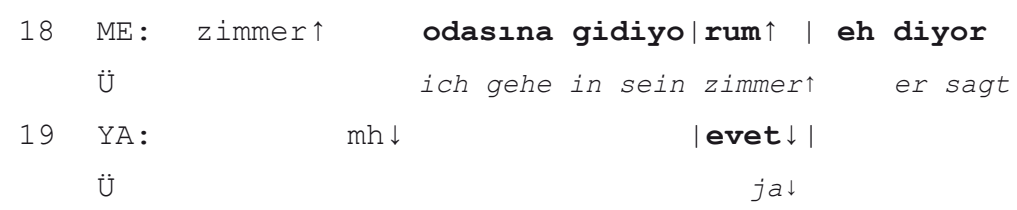

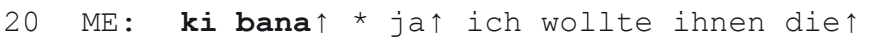
$\ddot{\mathrm{U}} \quad z u \operatorname{mir} \uparrow$

$21 \mathrm{ME}$ : mi"tteilung machen äh dass wir ihr gehalt

22 ME: erhöhen $\downarrow$ ben- |\#ach ja个\# LACHT| mir ging=s

Ü ich-

K \#LACHEND\#

23 YA: |oh: ja ja ja jal

$24 \mathrm{ME}$ : dann so \#richtisch gu:t $\uparrow$ ich hab alles\# K \#LEICHT LACHEND \#
25 ME: schme"rzen ver|gessen $\downarrow \mid$
26 YA: |millete| bak ya $\downarrow$ ee: niye
Ü schau dir die Leute an, Mensch $\downarrow$

27 YA: ge/ niye- has=du nich gesagt warum $\uparrow$

Ü und: warum ge/ warum-

$28 \mathrm{ME}: \quad<w i e s o$

29 ME: warum $\uparrow$ ich=ab=s verdie"nt $\downarrow>$ öyle şey sorulur $\ddot{U} \quad$ fragt man denn so

$30 \mathrm{ME}: \mathbf{m u} \downarrow \mid \leftarrow$ sanki ke"ndin| kendine zweifeln

$\ddot{U} \quad$ etwas $\downarrow$ als ob du an dir selbst zweifeln

31 YA: |LACHT 


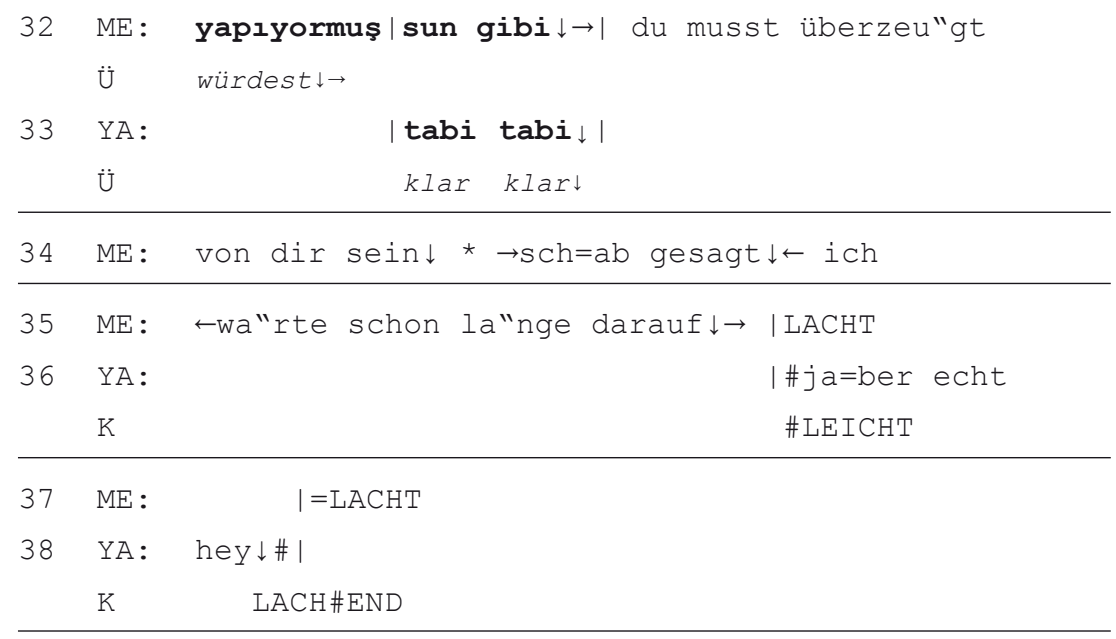

Der Transkriptausschnitt zeigt eindrucksvoll, wie häufig die Beteiligten in solchen Verdichtungspassagen zwischen Deutsch und Türkisch alternieren. Sprachwechsel finden zwischen allen möglichen sprachlichen Einheiten statt, zwischen und innerhalb von einzelnen Redebeiträgen und sogar innerhalb eines Wortes. In solchen Passagen ist es nicht möglich, eine Interaktionssprache zu identifizieren. D.h. für die Beteiligten stellt in solchen sprachlichen Verdichtungspassagen die ständige Alternation zwischen Deutsch und Türkisch den Interaktionscode selbst dar, sodass man in Auers (1999) Kategorien von einem Mischcode oder von der Praxis des Code-Mixings sprechen kann. Andererseits zeigt aber die gesprächsanalytische Untersuchung des Ausschnitts, dass viele Sprachalternationen sequenziell nicht völlig funktionslos sind. Bei der folgenden Analyse des Transkripts gehe ich chronologisch vor:

\section{Sprachwechsel zur Kontrastierung unterschiedlicher Interaktionsaktivitäten:}

Noch vor dem Transkriptausschnitt hatten die Beteiligten ein scherzhaftes Gespräch über MEs Beziehung zu BO, einem nicht anwesenden Gruppenmitglied, geführt. Mit MEs Äußerung in Zeile 01-02, $\leftarrow$ BO benim platonik askerm ama biç kimse bilmiyo bunu $\rightarrow$ simdilik- (BO ist meine platonische Liebe aber niemand weiß das $\downarrow$ bis jetzt-), wird dieses Thema beendet. In Zeile 02-03 initiiert ME einen neuen thematischen Strang. Sie bittet YA, der gerade das Fenster aufmachen will, weil die Luft im Clubraum stickig ist, das nicht zu tun: YA bitte nicht aufmachen $\downarrow$. Der Themawechsel wird von ME durch einen Sprachwechsel ins Deutsche markiert. 


\section{Sprachwechsel zur Aushandlung von Positionen:}

Die in Deutsch formulierte Bitte MEs, YA solle das Fenster nicht aufmachen, löst in der Folge eine Aushandlungssequenz aus. YA reagiert in Zeile 04-05 mit einem deutsch-türkischen Turn, in dem er zunächst auf Türkisch seinen Dissens andeutet, vallabi (wirklich), und dann auf Deutsch seine Gegenposition vorträgt: es=s doch hier (stickig). Nachdem ME auf ihrer Position insistiert, die sie erneut auf Deutsch formuliert, es war so angenehm so warm $\downarrow$ (Z. 07), reagieren nun sowohl YA (Z. 05-08) als auch TU (Z. 09) mit türkisch formulierten Gegenargumenten. Die Aushandlungssequenz kommt zum Abschluss, als ME in Zeile 10-11 ihre Position aufgibt und auch sprachlich mit YA und TU konvergiert, indem sie ihre Zustimmung auf Türkisch formuliert: iyi hadi çısin $\downarrow$ (gut, dann soll die [stickige Luft] halt raus).

\section{Sprachwechsel zur Ankündigung und Binnenstrukturierung von Erzählungen:}

Nach ihrer Zustimmung und einer kurzen Pause kündigt ME in Zeile 11-12 eine Erzählung an und wechselt dazu vom Türkischen ins Deutsche: <hey $\uparrow$ beute war ich wieder in frankfurt den ganzen tag ne个. Die Erzähleinleitung in Zeile 14 ist dann im deutsch-türkischen Mischcode ja: orda bizim bereichsleitungu görïyorum (ja, dort sehe ich unsere Bereichsleitung). ${ }^{17}$ Nachdem sie in Zeile 15 auf Türkisch eine Hintergrundinformation liefert, böyle çok önemli yukarlardan bi adam $\downarrow$ (so ein sehr wichtiger $\downarrow$ ein Mann von den Oberen $\downarrow$ ), wechselt sie bei der Einleitung und Wiedergabe der direkten Rede (des Bereichsleiters) erneut ins Deutsche: (er) bat gesagt- kommen sie kurz in mein zimmer $\uparrow$ (Z. 1518). Für die Darstellung der Szene setzt ME die beiden Sprachen funktional ein: Sie gibt die Zitate auf Deutsch wieder und formuliert ihre Rahmungen auf Türkisch. Wie routinisiert dieses rhetorische Muster in hoher Erzählgeschwindigkeit gebraucht wird, verdeutlicht vor allem in Zeile 22 die elliptische türkische Rederahmung, ben (ich), der ohne Pause deutsche Zitate vorangehen und folgen. Schließlich beendet ME ihre Erzählung mit einem Kommentar, der wie die Erzählankündigung auf Deutsch formuliert ist und wie eine abschließende Rahmung funktioniert: mir ging $=s$ dann so richtisch gu: $t \uparrow$ ich hab alles schme "rzen vergessen (Z. 22-25). Des Weiteren sind in dieser Passage insbesondere die Rezipientensignale von YA (Z. 17 und Z. 19) hervorzuheben. In bemerkenswerter Weise sind seine Reaktionen sprachlich mit den Äußerungen von ME synchronisiert: Parallel zu MEs deutscher Redewiedergabe sagt er echt $\uparrow$ (Z. 17) und kurze Zeit später parallel zu ihrer

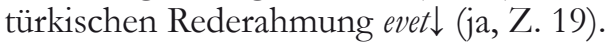

$17 \quad$ Zur genauen Analyse dieses Mixing-Musters siehe weiter unten. 


\section{Sprachwechsel zur Markierung eines Kommentars:}

YAs Reaktion auf MEs Erzählung nach ihrem Kommentar ist emotional distanziert. Der Wechsel der Äußerungsmodalität von, euphorisch' (ME) zu ,distanziert' (YA) wird zusätzlich durch einen Sprachwechsel ins Türkische hervorgehoben: millete bak ya (schau dir die Leute an, Mensch $\downarrow$, Z. 26). Nach dieser formelhaften Wendung bleibt YA im Türkischen und setzt zu einer Frage an, niye ge/ niye- (warum ge/ warum-), die er abbricht und auf Deutsch reformuliert: has=du nicht gesagt warum $\uparrow$ (Z. 26-27). Über die Gründe des Abbruchs und der Reformulierung kann hier nur spekuliert werden. Eine plausible Erklärung ist, dass in YAs Frage ein (fiktives) Zitat eingebaut ist, das in der Referenzwelt auf Deutsch hätte formuliert werden müssen (ME gegenüber ihrer deutschen Bereichsleitung). Da aber YA das im Zitat zentrale Fragewort „warum“ zunächst auf Türkisch formuliert, niye (warum), könnte für ihn der Grund dafür gewesen sein, die gesamte Äußerung abzubrechen und auf Deutsch zu reformulieren. Eine andere Erklärung wäre, dass sich YA so sehr im bilingualen Modus befindet, dass er in selbstverständlicher Weise lokale Prozessierungsprobleme durch Wechsel und Reformulierung in der anderen Sprache löst.

\section{Sprachwechsel zur Markierung von Argumentationsmustern:}

ME reagiert auf YAs distanzierte und hinterfragende Äußerung ausgesprochen erbost. Laut und mit dem Argumentationsmuster „Gegenfrage + Antwort" widerspricht sie YA in Zeile 28-29: <wieso warum $\uparrow$ ich $=a b=s$ verdie " $n t \downarrow>$. Dann expandiert und präzisiert sie ihre Position, verwendet dabei dasselbe Argumentationsmuster und hebt es zusäzlich durch einen Wechsel in den

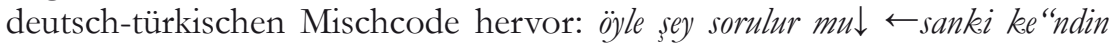
kendine zweifeln yapryormussun gibi $\downarrow \rightarrow$ (fragt man denn so etwas $\downarrow$ als ob du an dir selbst zweifeln würdest $\downarrow$, Z. 29-32). Mit dem anschließenden Wechsel ins Deutsche markiert ME die Schlussfolgerung ihrer Argumentation: $d u$ musst überzen"gt von dir sein $\downarrow * \rightarrow$ sch $=$ ab gesagt $\downarrow \leftarrow$ ich $\leftarrow$ wa"rte schon la "nge darauf $\downarrow \rightarrow($ Z. 32-35).

\section{Sprachübernahme zur Konsenshervorhebung:}

Betrachtet man YAs Reaktionen auf den letzten Redebeitrag von ME, so fällt seine sprachliche Synchronisierung ins Auge. ${ }^{18}$ In Zeile 33 reagiert er parallel zu MEs Äußerung, deren Matrixsprache Türkisch ist, ebenfalls auf

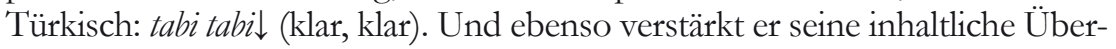

18 In bilingualen Kommunikationen ist häufig zu beobachten, dass die Beteiligten Konsens mit Sprachübernahme und Dissens mit unterschiedlicher Sprachverwendung anzeigen (siehe zum Beispiel in Keim (2007, S. 366-379) zu Sprachwechselmuster bei Aushandlungen und Streit). 
einstimmung in Zeile 36-38 durch Übernahme von MEs Interaktionssprache (Deutsch): ja=aber echt hey $\downarrow$. Mit dieser fein abgestimmten Sprachwahl unterstreicht er in höchst effektiver Weise seinen Konsens mit ME.

Neben diesen sequenziell bedeutungsvollen Sprachwechseln zeigen insbesondere zwei Äußerungen, welche Muster im Code-Mixing für die „Unmündigen" charakteristisch sind. Dabei handelt es sich um die Formulierungen in Zeile 14, ja: orda biəzim bereichsleitungu görïyorum $\uparrow$ (ja: dort sehe ich unsere Bereichsleitung $\uparrow$ ), und in Zeile 30-32, sanki kendin kendine zweifeln yapıyormussun gibi (als ob du an dir selbst zweifeln würdest):

a) $\operatorname{ME~(Z.~14):~ja:~orda~biz̨im~bereichsleitungu~görïyorum~} \uparrow$ (ja: dort sehe ich unsere Bereichsleitung $\uparrow)$ :

Die Matrixsprache der Äußerung ist Türkisch. Sie beinhaltet aber die vorgeschaltete deutsche Interjektion „ja “ und die deutsche Insertion „Bereichsleitung", die mit dem adäquaten türkischen Akkusativsuffix -u versehen und somit in den Matrixsatz integriert ist. Dabei kann das Substantiv „Bereichsleitung“ aus der Perspektive der Sprecherin als ein „core borrowing“ (Myers-Scotton 1993a, S. 168 ff.) eingestuft werden, über dessen türkisches Äquivalent „bölüm şefi“ die Sprecherin verfügt. Der Grund für die Verwendung des deutschen Ausdrucks ist darin zu sehen, dass er im Kontext der Erzählung - ME arbeitete zum Zeitpunkt der Aufnahme in einer deutschen Bank - lebensweltlich naheliegender ist als der türkische.

b) ME (Z. 30-32): sanki kendin kendine żpeifeln yapiyormussun gibi (als ob du an dir selbst zweifeln würdest):

Auch diese Formulierung ist ein Beleg für deutsch-türkisches Code-Mixing. In die türkische Äußerung ist das deutsche Verb „Zweifeln“ insertiert, das mit Hilfe des direkt folgenden flektierten türkischen Tunverbs „yapmak“ in die Matrixsprache integriert wird. ${ }^{19}$ Neben der Tatsache, dass die Sprecherin auch in diesem Fall über den türkischen Ausdruck für „Zweifeln“ (şüphelenmek) verfügt, ist insbesondere die aus lebensweltlicher Perspektive geringe semantische Spezifität des Verbs „zweifeln“ (im Gegensatz zu „Bereichsleitung“ oben) ein Argument dafür, dass es sich hierbei um die Variationspraxis des Code-Mixings handelt. ${ }^{20}$ Dabei zeigt das Reflexivpronomen „kendine“ (mit dem Dativsuffix „,-(n)e"), dass im Mischcode nicht alles grammatisch wohlgeformt sein muss/ist bzw. die Grammatiken beider

19 Backus (1996, S. 230-283) untersucht anhand von niederländisch-türkischen Variationsfällen sehr ausführlich und detailliert die Struktur ,niederländisches Verb im Infinitv + flektierte Form von yapmak“. Zu deutsch-türkischen Strukturen mit „yapmak“ siehe in Hinnenkamp (2005) und Keim (2007). Zur Integration von norwegischen Verben mit Hilfe des türkischen Tunverbs ,yapmak“ siehe in Türker (2000).

20 Zum Ansatz der „semantischen Spezifität“ von Einheiten beim Code-Switching siehe Backus (2001). 
Sprachen in eine interessante Interaktion treten. Die Formulierung mit dem türkischen Verb „şüphelenmek“ für „zweifeln“ hätte das Reflexivpronomen „kendinden“ mit dem Ablativsuffix ,-(n)den“ benötigt. Die Verwendung des deutschen Verbs „zweifeln“, das die Präposition „an“ mit folgendem Dativ(-Objekt) erfordert, scheint hier prospektiv bzw. regressiv die Auffälligkeit ausgelöst zu haben.

\section{Schluss}

Im vorliegenden Aufsatz wurde die Sprachorientierung und die Sprachvariationspraxis der Mannheimer Gruppe der „Unmündigen“ untersucht. Diese zeichnen sich durch zwei Aspekte aus: Einerseits ist die sprachliche Orientierung der Gruppe dadurch geprägt, dass Deutsch ihre unzweifelhaft dominante Interaktionssprache ist. Auf der anderen Seite kommt es in der gruppeninternen Kommunikation immer wieder zu Interaktionspassagen, in denen die Beteiligten zwischen Deutsch und Türkisch alternieren. In der Regel handelt es sich dabei um lokal bedeutungsvolles Code-Switching, d.h. um sequenziell kleinräumige Sprachalternation, der ansonsten lange deutsche Interaktionspassagen voran- und nachgehen. In anderen Fällen wechseln die Beteiligten über einen relativ längeren Zeitraum zwischen den beiden Sprachen, was ich als sequenziell großräumige Sprachalternation bezeichnet habe. Bei diesen Fällen von Code-Mixing sind die einzelnen Alternationen nicht mehr lokal bedeutungsvoll. Ihre Bedeutung ist auf einer globaleren Ebene zu sehen und zwar als Gruppencode.

Dieser Aspekt wird besonders dann deutlich, wenn man stilistische Befunde kontrastiert. In meiner Dissertation, in der ich das Sprachverhalten der „Unmündigen“ mit dem der „akademischen Europatürken“ vergleiche, zeigt sich, dass die dominante Interaktionssprache der „Europatürken“ Türkisch ist. Außerdem betrachten die „Europatürken“ die deutsch-türkische Variation, die auch bei ihnen zu beobachten ist, als Ausdruck von „doppelter Halbsprachigkeit ${ }^{\text {‘21 }}$, weshalb sie von ihnen dispräferiert und in der Interaktion durch sprachliche und metasprachliche Mittel markiert wird. Im Gegensatz dazu stellt die Sprachvariation bei den „Unmündigen“ einen wichtigen Ausdruck ihrer Identität als „emanzipatorische Migranten“ dar. Die Haltung der „emanzipatorischen Migranten“ gegenüber der Variationspraxis ist positiv. Es gibt im gesamten Material keine einzige Stelle, an der sie deutsch-türkische Sprachvariationen als dispräferiert markieren.

$21 \quad$ Der Begriff der „doppelten Halbsprachigkeit“ bzw. des „Semilingualismus“ wurde, wie Skutnabb-Kangas (1984, S. 248 ff.) ausführt, von Hansegård (1968) geprägt und meint in Bezug auf Migrantenkinder einen sprachlichen Zustand, der sich sowohl durch eine mangelhafte Beherrschung der Sprache der Herkunfts- als auch der Einwanderungsgesellschaft auszeichne. Siehe dazu auch in Cummins (1984). 


\section{Literatur}

Auer, Peter (1984): Bilingual conversation. (= Pragmatics and Beyond 5,8). Amsterdam/ Philadelphia.

Auer, Peter (1986): Kontextualisierung. In: Studium Linguistik 19, S. $22-47$.

Auer, Peter (1998): Introduction: Bilingual conversation revisited. In: Auer (Hg.), S. $1-$ 24.

Auer, Peter (Hg.) (1998): Code-Switching in conversation: language, interaction and identity. London/New York.

Auer, Peter (1999): From code-switching via language mixing to fused lects: toward a dynamic typology of bilingual speech. In: International Journal of Bilingualism 3, S. 309-332.

Backus, Albert Marie (1996): Two in one: bilingual speech of Turkish immigrants in the Netherlands. (= Studies in Multilingualism 1). Tilburg.

Backus, Albert Marie (2001): The role of semantic specificity in insertional codeswitching: evidence from Dutch-Turkish. In: Jacobson, Rodolfo (Hg.): Codeswitching worldwide. Bd. 2. Berlin/New York, S. 125-154.

Blom, Jan Petter/Gumperz, John Joseph (1972): Social meaning in linguistic structures: code-switching in Norway. In: Gumperz/Hymes (Hg.), S. 407-434.

Cassisi, Gaetano (2008): Ich Italiener, morgen andere Baustelle. Der lange Weg eines Emigranten nach Deutschland. (= Malstatter Beiträge aus Gesellschaft, Wissenschaft, Politik und Kultur). Merzig.

Cindark, Ibrahim (2010): Migration, Sprache und Rassismus. Der kommunikative Sozialstil der Mannheimer „Unmündigen“ als Fallstudie für die „emanzipatorischen Migranten“. (= Studien zur Deutschen Sprache 51). Tübingen.

Cummins, Jim (1984): Bilingualism and special education: issues in assessment and pedagogy. (= Multilingual Matters 6). Clevedon.

Dirim, İnci/Auer, Peter (2004): Türkisch sprechen nicht nur die Türken. Über die Unschärfebeziehung zwischen Sprache und Ethnie in Deutschland. (= Linguistik Impulse \& Tendenzen 4). Berlin/New York.

Ferguson, Charles A. (1959): Diglossia. In: Word. Journal of the Linguistic Circle of New York, 15, S. 325-340.

Fishman, Joshua A. (1965): Who speaks what language to whom and when? In: La Linguistique 2, S. 67-88.

Goffman, Erving (1978): Interaktionsrituale. Über Verhalten in direkter Kommunikation. (= Suhrkamp-Taschenbuch Wissenschaft 594). Frankfurt a.M.

Gumperz, John Joseph (1972): Introduction. In: Gumperz/Hymes (Hg.), S. 1-25.

Gumperz, John Joseph (1982): Discourse strategies. (= Studies in Interactional Sociolinguistics 1). Cambridge u.a.

Gumperz, John Joseph/Hymes, Dell (Hg.) (1964): The ethnography of communication. In: American Anthropologist 66, 6,2. 
Gumperz, John Joseph/Hymes, Dell (Hg.) (1972): Directions in sociolinguistics: the ethnography of communication. New York u.a.

Hansegård, Nils Erik (1968): Tvåspråkighet eller halvspråkighet? Med ett tillägg „De sprakliga minoritererna“ av Thomas Lundén. (= Aldusserien 254). Stockholm.

Hinnenkamp, Volker (2005): „Zwei zu bir miydi?““ - Mischsprachliche Varietäten von Migrantenjugendlichen im Hybriditätsdiskurs. In: Hinnenkamp, Volker/Meng, Katharina (Hg.): Sprachgrenzen überspringen. Sprachliche Hybridität und polykulturelles Selbstverständnis. (= Studien zur Deutschen Sprache 32). Tübingen, S. 51-103.

Kallmeyer, Werner/Schmitt, Reinhold (1996): Forcieren oder: Die verschärfte Gangart. Zur Analyse von Kooperationsformen im Gespräch. In: Kallmeyer, Werner (Hg.): Gesprächsrhetorik. Rhetorische Verfahren im Gesprächsprozeß. (= Studien zur Deutschen Sprache 4). Tübingen, S. 19-118.

Keim, Inken (1995): Kommunikative Stilistik einer sozialen Welt „kleiner Leute“ in der Mannheimer Innenstadt. (= Schriften des Instituts für Deutsche Sprache 4: Kommunikation in der Stadt 3). Berlin/New York.

Keim, Inken (2007): Die „türkischen Powergirls“. Lebenswelt und kommunikativer Stil einer Migrantinnengruppe in Mannheim. (= Studien zur Deutschen Sprache 39). Tübingen.

Keim, Inken/Cindark, Ibrahim (2003): Deutsch-türkischer Mischcode in einer Migrantinnengruppe: Form von "Jugendsprache“ oder soziolektales Charakteristikum? In: Neuland, Eva (Hg.): Jugendsprachen - Spiegel der Zeit. Internationale Fachkonferenz 2001 an der Bergischen Universität Wuppertal. (= Sprache, Kommunikation, Kultur 2). Frankfurt a.M. u.a., S. 377-394.

Labov, William (1971): The notion of ,system` in creole languages. In: Hymes, Dell (Hg.): Pidginization and creolization of languages. Proceedings of a conference, held at the University of the West Indies Mona, Jamaica, April 1968. Cambridge, S. $447-472$.

Myers-Scotton, Carol (1993a): Duelling languages: grammatical structure in codeswitching. Oxford.

Myers-Scotton, Carol (1993b): Social motivations for code-switching: evidence from Africa. (= Oxford Studies in Language Contact). Oxford.

Rampton, Ben (1995): Crossing: language and ethnicity among adolescents. (= Real Language Series). London u.a.

Schmitt, Reinhold (1993): Kontextualisierung und Konversationsanalyse. In: Deutsche Sprache 21, S. 326-354.

Sebba, Mark/Wootton, Tony (1998): We, they and identity: sequential versus identityrelated explanation in code-switching. In: Auer (Hg.), S. 262-289.

Skutnabb-Kangas, Tove (1984): Bilingualism or not: the education of minorities. (= Multilingual Matters 7). Clevedon.

Türker, Emel (2000): Turkish-Norwegian codeswitching. Evidence from intermediate and second generation Turkish immigrants in Norway. Oslo. 
Wei, Li (1998): The „why“ and „how“ questions in the analysis of conversational codeswitching. In: Auer (Hg.), S. 156-179.

Weinreich, Uriel (1953): Languages in contact: findings and problems. (= Publications of the Linguistic Circle of New York 1). New York.

Zifonun, Dariuš/Cindark, Ibrahim (2004): Segregation oder Integration? Die soziale Welt eines ,türkischen' Fußballvereins in Mannheim. In: Deutsche Sprache 32, S. 270-298. 
